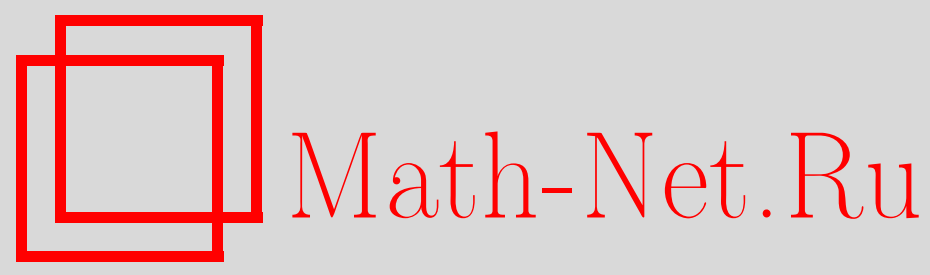

В. А. Демьяненко, О точных оценках кручения эллиптических кривых, Матем. заметки, 1998, том 63, выпуск 4, 503508

DOI: https://doi.org/10.4213/mzm1310

Использование Общероссийского математического портала Math-Net.Ru подразумевает, что вы прочитали и согласны с пользовательским соглашением http://www . mathnet.ru/rus/agreement

Параметры загрузки:

IP: 35.174 .16 .151

26 апреля 2023 г., 16:00:34

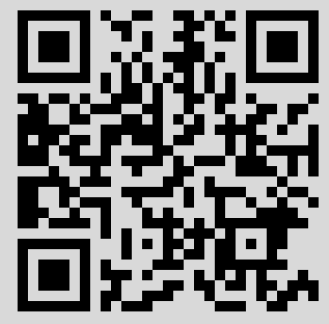




\section{О ТОЧНЫХ ОЦЕНКАХ КРУЧЕНИЯ ЭЛЛИПТИЧЕСКИХ КРИВЫХ}

\section{В. А. Демьяненко}

Над произвольным алгебраическим числовым полем указаны неулучшаемые оценки кручения кривых $y^{2}=x^{3}+s$ и $y^{2}=x^{3}+r x$.

Библиография: 3 названия.

Пусть $\mathbb{k}$ - алгебраическое числовое поле степени $n$ и $F_{1}: y^{2}=x^{3}+s, F_{2}: y^{2}=x^{3}+r x-$ кривые, определенные над этим полем.

Цель настоящей заметки - доказательство теорем, приведенных в тезисах [1].

Пусть $O_{m}=\left\{x_{1 / m}, y_{1 / m}\right\}$ - примитивная $\mathbb{k}$-точка порядка $m$, расположенная на одной из кривых $F_{1}$ или $F_{2}$, и $t O_{m}=\left\{x_{t / m}, y_{t / m}\right\}$.

ЛЕмма 1. Если $O_{2^{\alpha}}-$ примитивная $\mathbb{k}-$ точка на $F_{1}$, то

$$
2^{2 \alpha-3} \leqslant n \text {. }
$$

ДокАЗАТЕЛЬСтво. Возьмем на $F_{1}$ точку третьего порядка $O_{3}=\left\{x_{1 / 3}, y_{1 / 3}\right\}=$ $\{0, \sqrt{s}\}$ и рассмотрим тождество

$$
\frac{x_{1 / 2^{\alpha}}^{2^{2 \alpha-2}}}{x_{1 / 2}}= \pm 2^{2\left(2^{2 \alpha-2}-1\right) / 3} \prod_{l=0}^{\alpha-2} z_{2^{l}}^{2^{2 l}}, \quad z_{2^{l}}=\frac{y_{1 / 2^{l+2}}^{2} y_{1 / 3}^{2}}{y_{1 / 2^{l+2}+1 / 3} y_{1 / 2^{l+2}-1 / 3}}
$$

вытекающее из соотношений

$$
\frac{\left(x_{2^{t-1} a}-x_{2^{t-1} b}\right)^{4}}{x_{2^{t} a}-x_{2^{t} b}}=-4 \frac{y_{2^{t-1} a}^{2} y_{2^{t-1} b}^{2}}{y_{2^{t-1}(a-b)} y_{2^{t-1}(a+b)}}, \quad t=1,2, \ldots .
$$

Имеем $y_{1 / 3}^{2}=s, y_{a+1 / 3} y_{a-1 / 3}=s\left(8 s-x_{a}^{3}\right) / x_{a}^{3}$, вследствие этого $z_{2^{l}} \in \mathbb{k}$. Так как $x_{1 / 2^{t}}=s^{1 / 3} x_{1 / 2^{t}}^{\prime}, x_{1 / 2}=s^{1 / 3}, y_{1 / 2^{t}}=s^{1 / 2} y_{1 / 2^{t}}^{\prime}(t>1)$, где $x_{1 / 2^{t}}^{\prime}, y_{1 / 2^{t}}^{\prime}$ - целые алгебраические числа, причем $\left(y_{1 / 2^{t}}^{\prime}, 2\right)=1$, получаем

$$
x_{1 / 2^{\alpha}}^{\prime}= \pm 2^{2\left(2^{2 \alpha-2}-1\right) / 3} z, \quad z \text { - целое }, \quad(z, 2)=1 .
$$

Переходя к сопряженным полям $\mathbb{k}^{(i)}(i=1,2, \ldots, n)$, из (4) имеем

$$
\frac{2}{3}\left(2^{2 \alpha-2}-1\right) n \equiv 0\left(\bmod 2^{2 \alpha-2}\right), \quad n \equiv 0\left(\bmod 2^{2 \alpha-3}\right) .
$$

Лемма доказана. 
ЛЕмма 2. Если $O_{3^{\beta}}-$ примитивная $\mathbb{k}-$ точка на $F_{1}$, то

$$
\begin{array}{ll}
3^{2 \beta-3} \leqslant n & \text { nри } x_{3^{\beta-1} / 3^{\beta}}=x_{1 / 3}=0 \\
3^{2 \beta-2} \leqslant n & \text { npu } x_{3^{\beta-1} / 3^{\beta}}=x_{1 / 3}=-\sqrt[3]{4 s} .
\end{array}
$$

ДокАЗАТЕЛЬСТво. Возьмем на $F_{1}$ точку второго порядка $O_{2}=\left\{x_{1 / 2}, y_{1 / 2}\right\}=$ $\{-\sqrt[3]{s}, 0\}$ и рассмотрим кривую

$$
v^{2}=u^{4}+3 \rho u^{2}+3 \rho^{2}, \quad \rho=-\sqrt[3]{s}, \quad u=\sqrt{x-\rho}, \quad v=\frac{y}{u} .
$$

На кривой (6) над полем $\mathbb{k}(\rho)$ расположена примитивная порядка $3^{\beta}$ точка $O_{3 \beta}=$ $\left\{u_{1 / 3^{\beta}}, v_{1 / 3^{\beta}}\right\}, u_{1 / 3^{\beta}}=\sqrt{x_{1 / 3^{\beta}}-\rho}, v_{1 / 3^{\beta}}=y_{1 / 3^{\beta}} / u_{1 / 3^{\beta}}$, поэтому $u_{1 / 3^{\beta}}, v_{1 / 3^{\beta}} \in \mathbb{k}(\rho)$. Для $u_{1 / 3^{\beta}}$ кривой $(6)$ имеем

$$
u_{1 / 3^{\beta}}= \begin{cases}3^{\left(1-3^{2 \beta-2}\right) / 4} s^{1 / 6} u_{1 / 3^{\beta}}^{\prime} & \text { при } x_{3^{\beta-1} / 3^{\beta}}=x_{1 / 3}=0, \\ 3^{\left(1-3^{2 \beta-1}\right) / 4} s^{1 / 6} u_{1 / 3^{\beta}}^{\prime} & \text { при } x_{3^{\beta-1} / 3^{\beta}}=x_{1 / 3}=-\sqrt[3]{4 s},\end{cases}
$$

где $u_{1 / 3^{\beta}}^{\prime}-$ алгебраическая единица.

Из (7) аналогично лемме 1 следует

$$
\begin{array}{ll}
n \equiv 0\left(\bmod 3^{2 \beta-3}\right) & \text { при } x_{1 / 3}=0, \\
n \equiv 0\left(\bmod 3^{2 \beta-2}\right) & \text { при } x_{1 / 3}=-\sqrt[3]{4 s} .
\end{array}
$$

Лемма доказана.

Лемма 3. Если $O_{p}$ - примитивная $\mathbb{k}-$ точка на $F_{1}(p=6 t+1-$ простое $)$, то

$$
\varphi\left(p^{\gamma}\right)=p^{\gamma-1}(p-1) \leqslant 3 n
$$

ЛЕмма 4. Если $O_{p \gamma}-$ примитивная $\mathbb{k}-$ точка на $F_{1}(p=6 t+5-$ простое $)$, то

$$
p^{2 \gamma-2}\left(p^{2}-1\right) \leqslant 6 n
$$

Доказательства лемм 3 и 4 приведены в [2].

ЛЕмма 5. Если $O_{2^{\alpha}}-$ примитивная $\mathbb{k}-$ точка на $F_{2}$, то

$$
\begin{array}{ll}
2^{2 \alpha-4} \leqslant n & \text { npu } x_{2^{\alpha-1} / 2^{\alpha}}=x_{1 / 2}=0 \\
2^{2 \alpha-3} \leqslant n & \text { npu } x_{2^{\alpha-1} / 2^{\alpha}}=x_{1 / 2}=\rho=\sqrt{-r} .
\end{array}
$$


ДокАЗАТЕЛЬСтво. Запишем тождество

$$
\begin{gathered}
\frac{\left(x_{1 / 2^{\alpha}}-x_{1 / 2^{\alpha-1}}\right)^{2^{2 \alpha-4}}}{x_{1 / 4}-x_{1 / 2}}= \pm 2^{2\left(2^{2 \alpha-4}-1\right) / 3} \prod_{l=0}^{\alpha-3} z_{2^{2^{l}}}^{2^{(\alpha-3-l)}} \\
z_{2^{l}}=\frac{y_{1 / 2^{\alpha-l}}^{2} y_{1 / 2^{\alpha-1-l}}^{2}}{y_{1 / 2^{\alpha-l}-1 / 2^{\alpha-1-l} y_{1 / 2^{\alpha-l}+1 / 2^{\alpha-1-l}}}}
\end{gathered}
$$

следующее, как и (2), из соотношений (3).

Так как $\left(i^{2}=-1\right)$

$$
x_{1 / 4}-x_{1 / 2}= \begin{cases}i \rho & \text { при } x_{1 / 2}=0, \\ 2^{1 / 2} \rho & \text { при } x_{1 / 2}=\sqrt{-r}\end{cases}
$$

и

$$
y_{1 / 2^{t}}=2^{1 / 2} r^{3 / 4} y_{1 / 2^{t}}^{\prime}, \quad t>1,
$$

где $y_{1 / 2^{t}}^{\prime}$ - алгебраические единищы, из (11) и (12), как и в лемме 1, получаем

$$
\begin{aligned}
\left(2^{2 \alpha-4}-1\right) n & \equiv 0\left(\bmod 2^{2 \alpha-4}\right) & \text { при } x_{1 / 2}=0, \\
n+2\left(2^{2 \alpha-4}-1\right) n & \equiv 0\left(\bmod 2^{2 \alpha-3}\right) & \text { при } x_{1 / 2}=\sqrt{-r},
\end{aligned}
$$

и неравенства (10) справедливы.

Так как для кривой $F_{2}$

$$
\sqrt{x_{1 / p^{\gamma}}}=\frac{\left(x_{\left.(t / p)^{\gamma}-\rho\right)^{2}+2 \rho^{2}}\right.}{2 y_{(t / p)^{\gamma}}}, \quad 2 t \equiv 1\left(\bmod p^{\gamma}\right),
$$

при нечетном $p$ имеем $x_{1 / p^{\gamma}}=x_{1 / p^{\gamma}}^{\prime 2}, x_{1 / p^{\gamma}}^{\prime} \in \mathbb{k}$, и на основании [2] справедливы следующие леммы.

Лемма 6. Если $O_{p} \gamma-$ примитивная $\mathbb{k}-$ точка на $F_{2}(p=4 t+1-$ простое $)$, то

$$
\varphi\left(p^{\gamma}\right)=p^{\gamma-1}(p-1) \leqslant 2 n .
$$

ЛЕмма 7. Если $O_{p \gamma}-$ примитивная $\mathbb{k}-$ точка на $F_{2}(p=4 t+3-$ простое $)$, то

$$
p^{2 \gamma-2}\left(p^{2}-1\right) \leqslant 4 n
$$

Легко установить, что для некоторых кривых $F_{1}$ и $F_{2}$ в $(1),(5),(8)-(10),(13)$ и $(14)$ равенства достижимы, поэтому полученные оценки $p$-кручения являются уже неулучшаемыми. В частности, если $\mathbb{k}=\mathbb{Q}$ - поле рациональных чисел и $O_{m}$ - примитивная $\mathbb{Q}$-точка порядка $m$, то из лемм $1-4$ и лемм $5-7$ соответственно следует, что $6 \equiv 0(\bmod m)$ для кривой $F_{1}$ и $4 \equiv 0(\bmod m)$ для кривой $F_{2}$, т.е. получаем известную теорему Нагеля-Лутц-Морделла. 
ЛЕмма 8. Если

$$
m=m_{k}=\prod_{k=1}^{l} p_{k}^{\gamma_{k}}
$$

$\left(p_{k}=6 t_{k}+1-\right.$ простые) $и O_{m}-$ примитивная $\mathbb{k}-$ точка на $F_{1}$, то

$$
\varphi(m)=\varphi\left(m_{k}\right)=\prod_{k=1}^{l} p_{k}^{\gamma_{k}-1}\left(p_{k}-1\right) \leqslant 3 n .
$$

ДокАЗАтельство. Расширим поле $\mathbb{k}$ до $\mathbb{k}^{\prime}=\mathbb{k}(\sqrt[6]{s})$ и перепишем кривую $F_{1}$ над полем $\mathbb{k}^{\prime}$ в виде $y^{2}=x^{3}+1$. На основании лемм 6 и 10 работы [3] имеем

$$
\begin{gathered}
F_{1}^{\prime}: Y^{2}=X^{3}+r_{(\alpha / m)} X+s_{(\alpha / m)}, \\
r_{(\alpha / m)}=-15 \sum_{\delta=1}^{m-1} x_{\delta(\alpha / m)}^{2}, \quad s_{(\alpha / m)}=s-7 \sum_{\delta=1}^{m-1}\left(2 s+5 x_{\delta(\alpha / m)}^{2}\right),
\end{gathered}
$$

$P$ - произвольная $\mathbb{k}$-точкана $F_{1}$ и $\left\{O_{m}, O_{m}^{\prime}\right\}$ - базис всех точек порядка $m$ на $F_{1}$,

$$
\begin{gathered}
\alpha_{1} O_{m}+\alpha_{2} O_{m}^{\prime}=\left\{x_{(\alpha / m)}, y_{(\alpha / m)}\right\}, \quad\left(\alpha_{1}, \alpha_{2}, m\right)=1, \\
P+\alpha_{1} O_{m}+\alpha_{2} O_{m}^{\prime}=\left\{x_{1,(\alpha / m)}, y_{1,(\alpha / m)}\right\} \\
P^{\prime}=\left\{X_{1,(\alpha / m)}, Y_{1,(\alpha / m)}\right\}=\left\{\sum_{\gamma=0}^{m-1} x_{1, \gamma(\alpha / m)}-\sum_{\delta=1}^{m-1} x_{\delta(\alpha / m)}, \sum_{\gamma=0}^{m-1} y_{1, \gamma(\alpha / m)}\right\} \in F_{1}^{\prime}
\end{gathered}
$$

и

$$
\begin{gathered}
P=\beta_{1} O_{m}+\beta_{2} O_{m}^{\prime}=\left\{x_{(\beta / m)}, y_{(\beta / m)}\right\}, \quad(\beta / m) \neq l(\alpha / m), \quad\left(\beta_{1}, \beta_{2}, m\right)=1, \\
X_{t(\beta / m),(\alpha / m)}=\sum_{k=0}^{[m / 2]} a_{k,(\alpha / m)}\left(\varepsilon^{t}-\varepsilon^{-t}\right)^{2 k-2}, \\
Y_{t(\beta / m),(\alpha / m)}=\sum_{k=0}^{[m / 2]} b_{k,(\alpha / m)}\left(\varepsilon^{t}-\varepsilon^{-t}\right)^{2 k-3}, \\
\varepsilon=e^{2 \pi i / m}, \quad a_{k,(\alpha / m)}, b_{k,(\alpha / m)} \in \mathbb{Q}\left(s_{k}\right), \quad s_{k}-\text { симметрические функции, } \\
\sum_{\delta_{1}, \ldots, \delta_{k}} x_{\delta_{1}(\alpha / m)} \cdots x_{\delta_{k}(\alpha / m)} .
\end{gathered}
$$

Если $\varphi(m)>3 n$, то из (15) получаем

$$
D_{(\alpha / m)}=\frac{D^{m}}{\prod_{\delta=1}^{m-1} y_{0, \delta(\alpha / m)}^{4}}=0, \quad D=0,
$$

что противоречит условию $D=-27 s^{2} \neq 0$. Лемма доказана.

Пусть

$$
m_{j}=\prod_{j=1}^{l} p_{j}^{\delta_{j}}
$$

$\left(p_{j}=6 t_{j}+5-\right.$ простые $)$ и

$$
\psi\left(m_{j}\right)=\prod_{j=1}^{l} p_{j}^{\delta_{j}-1}\left(p_{j}+1\right)
$$


Лемма 9. Если $m=m_{j}$ и $O_{m}-$ примитивная $\mathbb{k}-$ точка на $F_{1}$, то

$$
\varphi\left(m_{j}\right) \psi\left(m_{j}\right)=\prod_{j=1}^{l} p_{j}^{2 \delta_{j}-2}\left(p_{j}-2\right) \leqslant 6 n .
$$

ДокАЗАТЕЛьСтво. Запишем, как и в лемме 8, равенства

$\sum_{\{(\alpha / m)\}} X_{t(\beta / m),(\alpha / m)}=\sum_{k=0}^{[m / 2]}\left(\sum_{\{(\alpha / m)\}} a_{k,(\alpha / m)}\right)\left(\varepsilon^{t}-\varepsilon^{-t}\right)^{2 k-2}$ $(\beta / m) \notin\{(\alpha / m)\}$ $(\beta / m) \notin\{(\alpha / m)\}$

$$
\begin{aligned}
& =\sum_{\substack{k=0 \\
\{(\alpha / m)\}}}^{[m / 2]} a_{k,(\beta / m)}\left(\varepsilon^{t}-\varepsilon^{-t}\right)^{2 k-2}, \\
\sum_{t(\beta / m),(\alpha / m)} & =\sum_{k=0}^{[m / 2]}\left(\sum_{\substack{\{(\alpha / m)\} \\
(\beta / m) \notin\{(\alpha / m)\}}} b_{k,(\alpha / m)}\right)\left(\varepsilon^{t}-\varepsilon^{-t}\right)^{2 k-3} \\
& =\sum_{k=0}^{[m / 2]} b_{k,(\beta / m)}\left(\varepsilon^{t}-\varepsilon^{-t}\right)^{2 k-3} .
\end{aligned}
$$

Левые части равенств (17) есть рациональные функции от $x_{(\beta / m)}, y_{(\beta / m)}$, в то время как в правой части $a_{k,(\beta / m)} \in \mathbb{Q}\left(s_{k}\right), s_{k}$ - симметрические функции,

$$
\sum_{\delta_{1}, \ldots, \delta_{k}} x_{\delta_{1}(\beta / m)} \cdots x_{\delta_{k}(\beta / m)}
$$

Так как $\psi\left(m_{j}\right)$ - минимальная степень поля, которому принадлежат $a_{k,(\beta / m)}$, из $(17)$ следует (16).

Аналогично доказываются следующие утверждения.

ЛЕмма 10. Если

$$
m=m_{k}=\prod_{k=1}^{l} p_{k}^{\gamma_{k}}
$$

$\left(p_{k}=4 t_{k}+1-\right.$ простые) $и O_{m}-$ примитивная $\mathbb{k}$-точка на $F_{2}$, то

$$
\varphi(m)=\varphi\left(m_{k}\right)=\prod_{k=1}^{l} p_{k}^{\gamma_{k}-1}\left(p_{k}-1\right) \leqslant 2 n .
$$

ЛЕМма 11. Если

$$
m=m_{j}=\prod_{j=1}^{l} p_{j}^{\delta_{j}}
$$

$\left(p_{j}=4 t_{j}+3-\right.$ простые $)$ и $O_{m}-$ примитивная $\mathbb{k}-$ точка на $F_{2}$, то

$$
\varphi\left(m_{j}\right) \psi\left(m_{j}\right)=\prod_{j=1}^{l} p_{j}^{2 \delta_{j}-2}\left(p_{j}^{2}-1\right) \leqslant 4 n .
$$

Из лемм 1-4, 8, 9 и лемм 5-7, 10, 11 соответственно вытекают следующие теоремы. 
Теорема 1. Пусть $m=2^{\alpha} 3^{\beta} m_{k} m_{j}$ и $O_{m}-$ примитивная $\mathbb{k}-$-олкана $F_{2}$. Тогда

$$
2^{2 \alpha-3} 3^{2 \beta-3} \varphi\left(m_{k} m_{j}\right) \psi\left(m_{j}\right) \leqslant 6 n
$$

Теорема 2. Пусть $m=2^{\alpha} m_{k} m_{j} u O_{m}$ - примитивная $\mathbb{k}-$ точкана $F_{2}$. Тогда

$$
2^{2 \alpha-4} \varphi\left(m_{k} m_{j}\right) \psi\left(m_{j}\right) \leqslant 4 n \text {. }
$$

\section{СПИСОК ЦИТИРОВАННОЙ ЛИТЕРАТУРЫ}

[1] Демьяненко В.А. О точных оценках кручения эллиптических кривых // Третья международная конференция по алгебре. Тезисы докл. Красноярск, 1993. С. 106.

[2] Демьяненко В. А. О точных оценках $p$-кручения некоторых кривых первого рода // Матем. заметки. 1977. Т. 21. №1. С. 3-7.

[3] Демьяненко В.А. О точньх оценках кручения точек кривых первого рода // Записки научн. семин. ЛОМИ. 1986. Т. 151. С. 57-65.

Институт математики и механики УрО РАН 